\title{
Hydro-elastic response of composite hydrofoil with FSI
}

\author{
Temtching Temou Vanilla ${ }^{1,2,{ }^{*}}$, Augier Benoit ${ }^{3,}{ }^{*}$, Paillard Benoit 4
}

1 SEAIR, hydrofoil Resource Center, 10 rue chalutier les 2 anges, 56100 Lorient, France

2 Institut de Recherche de l'Ecole Navale, 29240 Brest Armées, France

3 IFREMER, Marine Structure Laboratory, 1625 Route de Sainte-Anne, 29280 Plouzané, France

${ }^{4}$ Alternative Current Energy, 35B Rue de Marmande, 33800 Bordeaux, France

* Corresponding authors : Vanilla Temtching Temou, email address : vanilla@seair.fr ; Benoit Augier, email address : benoit.augier@ifremer.fr

\begin{abstract}
:
The present study investigates experimentally and numerically the impact of composite materials on hydro-elastic performances of a hydrofoil experiencing Fluid-Structure Interactions, and focus on the bend-twist coupling phenomenon. Four flexible hydrofoils piercing the free surface, with identical geometry of extruded plan-form, a constant NACA0015 section, are tested in a cantilevered configuration in a hydrodynamic flume. The hydrofoils are built from the same mold with different materials (carbon or glass fiber) and different layups. The layups are designed to allow or not bend-twist coupling by the use of $45^{\circ}$ plies in the structure. Two different coupled FSI numerical approaches are developed to model the hydrofoils behavior: a low fidelity code based on the coupling of a Vortex Lattice Method and a beam theory and a high fidelity code made of the coupling of the structural model code-ASTER and an OpenFOAM VoF hydrodynamic model with free surface. Mechanical characterization of the hydrofoils highlights the differences on the structures which are exacerbated in the hydrodynamic tests. The bendtwist coupling induces a modification of the angle of attack at the tip, leading to a significant difference of the generated lift and thus the deformation. The bend-twist coupling and the hydrodynamic performances are simulated by the numerical approaches.
\end{abstract}

\section{Highlights}

- Numerical and experimental study of a composite hydrofoil. Bend-twist coupling of composite structure. FSI coupling on composite structure.

Keywords : Bend-twist coupling, Composite materials, Fluid-Structure Interactions, Hydrofoils 


\section{Introduction}

For the high performances foiling yachts, the design process of lifting surface is a complex combination of free surface hydrodynamic simulations coupled with structural calculation of highly loaded composite structures. The FluidStructure Interaction [FSI] must be considered in the design of yacht hydrofoils due to the significant deformation of the structure which impacts the hydrodynamics [4] and the yacht equilibrium [21] as illustrated in figure 1.

Within the available literature, a part of the described problem has been studied as the performance of a surface piercing straight hydrofoil [43] or the coupled effect of fluid-structure interactions on cavitation [14]. Ducoin [13] investigated numerically and experimentally the behavior of a flexible hydrofoil in a flow. Gaugain [16] studied numerically and experimentally a flexible NACA66-312 (mod.) hydrofoil in poly acetate with a coupled approach in a partitioned coupling.

Composite structures are extensively used for the appendages in the sailing yacht domain, putting more complexity in their behavior prediction. The structural behavior of a hydrofoil can greatly impact of the flow around it by increasing or reducing its hydrodynamic loading. Indeed, the anisotropy of composites materials can introduce extension-twist coupling or bend-twist coupling in the composite structure (see figure 2).

This phenomenon can reduce the incidence of the hydrofoil when the hydrodynamic load increases which can be a

${ }^{\star}$ This document is the results of the researches performed by the first author in its PhD work, granted by the hydrofoil resource center SEAIR.

*Principal corresponding author: benoit.augier@ifremer.fr

* Corresponding author: vanilla@ seair.fr

ORCID(s):

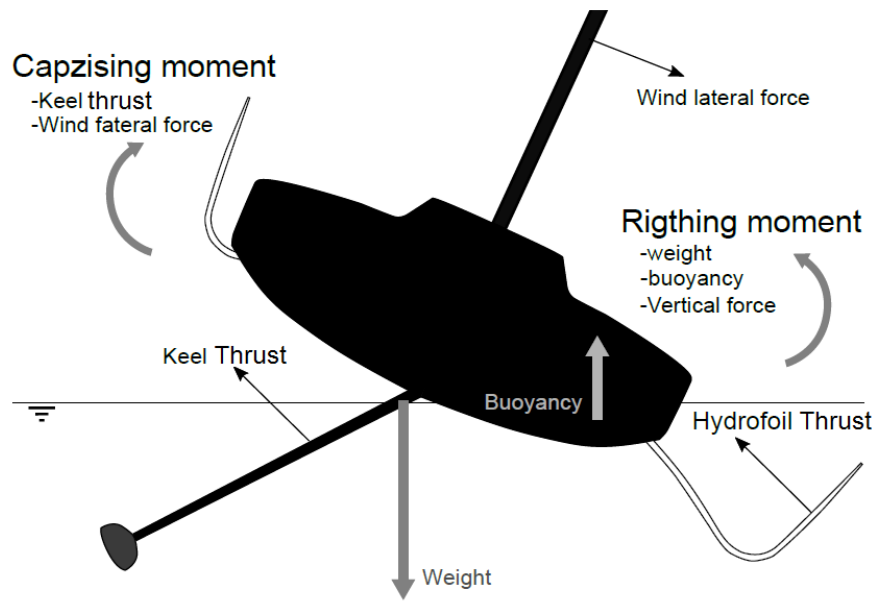

Figure 1: Modern 60' sailing yacht equilibrium [38].
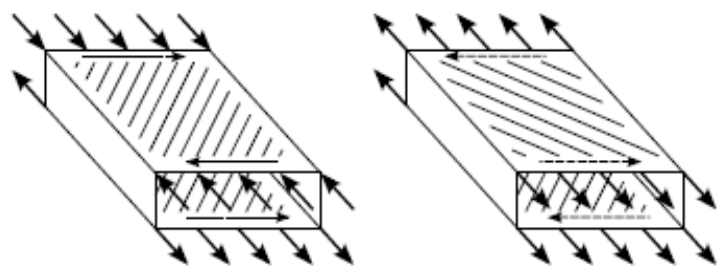

Figure 2: Bend-twist coupling (left) and extension-twist coupling (right) in beam composite structures. [15]

good approach to control the lifting force through FSI with a passive adaptive method. [23] tested various orientations in the laminate layup of a blade to study the effect of bend-twist 
coupling [BTC] on a wind turbine efficiency and came out with an increase of $10 \%$ in the wind turbine efficiency without changing the velocity nor the angle of attack. Manudha [30] proposed a Differential Stiffness Bend-Twist (DSBT) concept to achieve bend-twist coupling in the global structure of turbine blades and it shows good results in the control of the blade deformation behavior. [29], [5], [28], [39] performed several studies on load alleviation of wind turbine blades with BTC. Fatigue load reduction on wind turbines in a range of 10-20\% were highlighted with twist to feather coupling. The coupling phenomenon depends on the plies orientations in the layup. [37], [7] evaluated the percentage of bend-twist coupling with the plies orientation for glass and carbon fibers. Regarding hydrofoil applications, [17] has recently described the use of different techniques for bendtwist coupling as Passive Adaptive Composites (PAC) that tailors the response of a structure by changing the orientation of the composite plies and, DSBT that utilizes the internal stiffness of a structure to change the aero-hydrodynamic response to fluid load [33]. They have shown that the PAC can be used to control the lift response to hydrodynamic load in the case of a composite structure [18] mainly by decreasing the tip load. [25] numerically studied the influence of plies orientation on vibration and bend-twist coupling as well as the effect of anysotropy and sweep [27]. Experimental investigations were also carried out on the steady-state fluid-structure interactions of flexible composite hydrofoils ([46],[32]). More recently [44] and [26] studied the effect of bend-twist coupling on the hydro-elastic response of a composite hydrofoil in a cavitation tunnel and [20] looked at the FSI of a composite hydrofoil in forced rotational oscillation. [41] used multidisciplinary design optimisation to minimize the drag of an hydrofoil based on the outer shape and composite layout. Eventually, [31] investigated numerically and experimentally the fluid-structure interactions on a flexible composite hydrofoil under viscous flows and show a very strong hydro-elastic response, with a structural resonance due to the high flexibility of the structure.

The present study investigates experimentally and numerically the impact of composite layups on the hydrodynamic performances of a hydrofoil piercing the free surface in the case of large displacements at the tip (up to 10\% of the span). Modern sailing yacht foiling as the IMOCA 60' are now equipped with appendages that experience such deformations. These appendages, key point of the yacht equilibrium (figure 1), are extensively built in composite materials which allow the best compromise between strength and weight. The hydrodynamics loads experienced by the hydrofoils have reached a level where the optimization of the layup and the fabrication process of the composite has to be carefully study. As a first approach, the problem is simplified by a hydrofoil piercing the free surface at $45^{\circ}$. Four composite hydrofoils made up of carbon or glass fibers are built for the experiments. The numerical analysis is performed with two FSI open-source codes developed with a partitioned coupling approach. The first is a low fidelity code based on the coupling of a potential flow solver based on the vortex lat- tice method and a finite elements solver based on the beam theory. The second is a high fidelity code made of the coupling of the structural code ASTER and an OpenFOAM VoF hydrodynamic model with free surface. The second section of the paper describes the hydrofoils, their geometry and the different composite layups. The experimental setup of the static and experimental tests are also presented. The third section presents the numerical approaches to solve the fluid-structure interactions on the hydrofoils and the modeling of the bend-twist coupling is discussed. The fourth section presents the experimental results and a comparison to the simulations performed with the two codes. The principal objective of this paper is first to quantify and measure the effect of composite layup in bend-twist coupling appearance and therefore the impact on hydrodynamic performances of the hydrofoils and secondly to validate the numerical tools on composite hydrofoils.

\section{Hydrofoils description and experimental setup}

The four tested hydrofoils and the experimental setup for the static and dynamic tests are presented in this section.

\subsection{Hydrofoil description}

The hydrofoils are manufactured at SEAir, the industrial partner of this research work. The four geometries are all identical: a plan-form hydrofoil of $1.35 \mathrm{~m}$ span with a NACA0015 profile of $0.114 \mathrm{~m}$ constant chord. The cross-section is sandwich structure made of a laminated composite skin and an a web in Airex material. The hydrofoils only differ in the materials of their skins and are built by contact moulding on a 2 pieces aluminium mold: composite fibers are first impregnated in an epoxy resin and put in contact with the mold. This operation is performed on the two side of the mold giving the suction side and the high pressure side of the hydrofoils. Then, the Airex web is glued on the inside the two laminated skin and trimmed off. A hydrofoil is made of the two assembled sides using the Spabond 345 glue.

Figure 3 presents the geometry of the hydrofoils showing the location of the Airex web in the section and the laminated skin. The figure also shows a base directly molded at the root of the hydrofoils in a rectangular mold. That base is used for the clamping system of the structures in the experimental setups.

\subsection{Hydrofoil Materials}

Three of the hydrofoils, $P_{1,2,3}$, are built in glass fibers and the fourth $P_{4}$ is in carbon fibers. The four different layups are given in table 1, the layup $P_{1}$ uses the Glass $s_{2}$ when layup $P_{2}$ and $P_{3}$ uses the Glass 1 . In the layup orientations, \pm 45 represents a bi-axial ply with fibers oriented in the both directions $-45^{\circ}$ and $45^{\circ}$. Thus, $\left[( \pm 45)_{2} / 0 /( \pm 45)_{2}\right]$ will defined in the article a layup composed of two bi-axial plies oriented at $\pm 45^{\circ}$, followed by a ply oriented at $0^{\circ}$ and two others biaxial plies oriented at $\pm 45^{\circ}$.

The mechanical properties of the materials are given in table 2. The thickness of the laminated skins are $1 \mathrm{~mm}$ for 


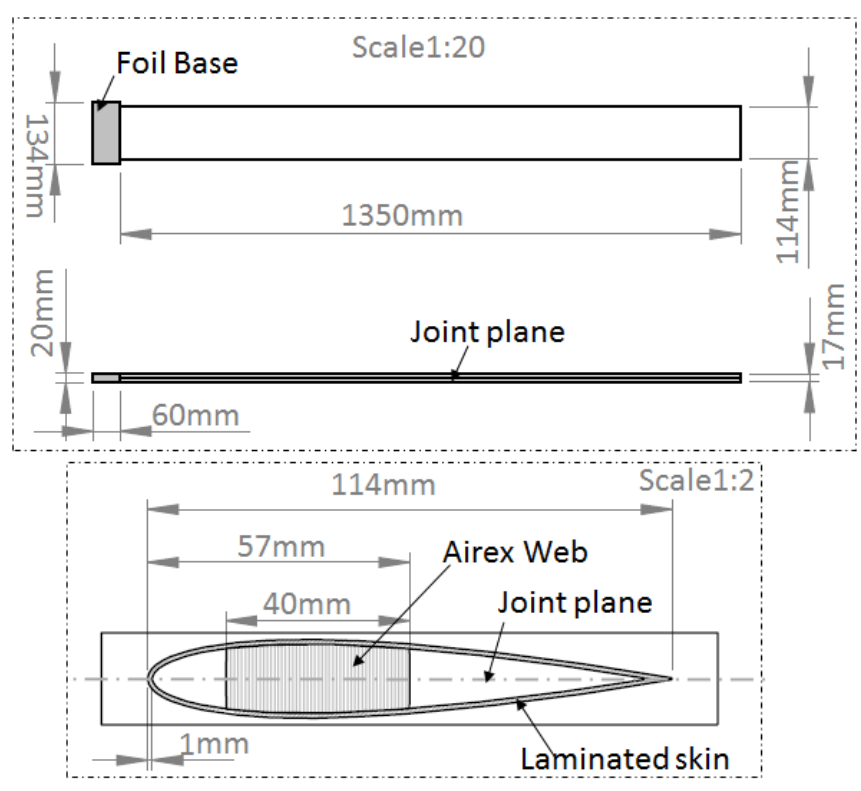

Figure 3: Hydrofoil description and geometrical parameters showing the Airex web location and the laminate skin of $1 \mathrm{~mm}$ thickness.

\begin{tabular}{|c|c|c|}
\hline Foils & Material & layup \\
\hline$P_{1}$ & Epoxy-Glass2 & {$\left[( \pm 45)_{2} / 0 /( \pm 45)_{2}\right]$} \\
\hline$P_{2}$ & Epoxy-Glass1 & {$[90 /-45 / 0 /-45 / 90]$} \\
\hline$P_{3}$ & Epoxy-Glass1 & {$[90 / 45 / 0 / 45 / 90]$} \\
\hline$P_{4}$ & Epoxy-Carbon & {$\left[90 /(0)_{2} / 90\right]$} \\
\hline
\end{tabular}

Table 1

Layups orientations of the four hydrofoils.

\begin{tabular}{|c|c|c|c|}
\hline Material & Carbon & Glass1 & Glass2 \\
\hline$E_{l}[G P a]$ & 120 & 54 & 45 \\
\hline$E_{t}[G P a]$ & 10 & 10.4 & 10 \\
\hline$G_{l t}[G P a]$ & 4 & 3.9 & 5 \\
\hline$v_{l t}$ & 0.362 & 0.25 & 0.25 \\
\hline
\end{tabular}

Table 2

Mechanical properties of the plies. Elastic modulus in the longitudinal $\left(E_{l}\right)$ and transverse $\left(E_{t}\right)$ direction. Shear modulus $G_{l t}$ and Poisson modulus $v_{l t}$.

the glass hydrofoils $P_{1,2,3}$ and $1.2 \mathrm{~mm}$ for the carbon hydrofoil $P_{4}$.

The results expected for these layup configurations are:

- $P_{1}$ : Important bending and no bend-twist coupling

- $P_{2}$ : Bend-twist coupling with a negative twist and higher bending when negative incidences are investigated

- $P_{3}$ : Bend-twist coupling with a positive twist and smaller bending when negative incidences are investigated

- $P_{4}$ : Small bending and no bend-twist coupling

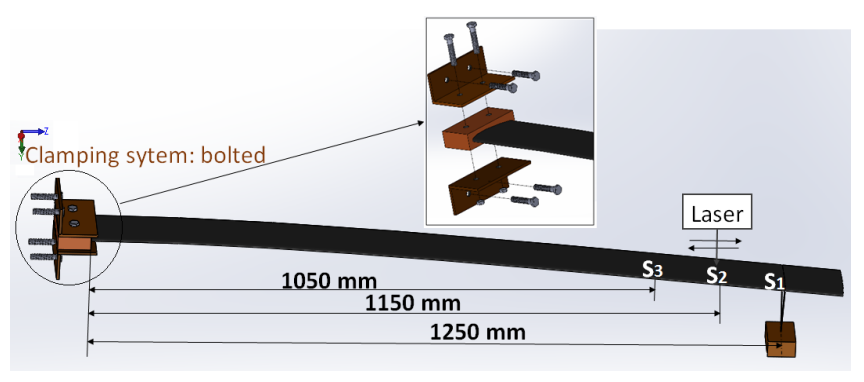

Figure 4: Experimental setup of the bending tests showing the clamping system, the loaded section $S_{1}$ and the measurement sections $S_{2}$ and $S_{3}$.

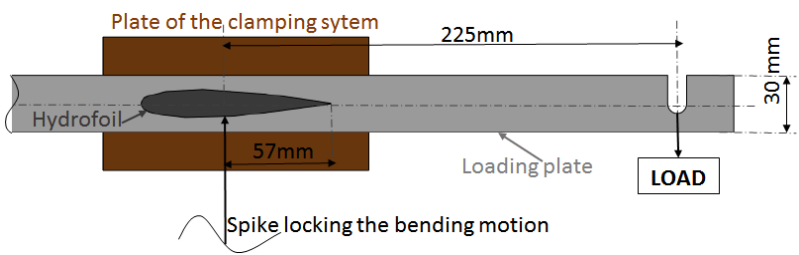

Figure 5: Loading method of the torsional tests, the loading plate is placed on the section $S_{1}(1250 \mathrm{~mm})$.

\subsection{Mechanical characterization}

This section describes the mechanical static tests performed in air to assess the bending and torsional stiffness of the hydrofoils as well as the BTC appearance. The experimental setup of the different tests is firstly described and the results are discussed.

\subsubsection{Experimental setup}

The experimental setups are presented in figures 4 and 5. The hydrofoils are mounted cantilevered-free and the detail of the clamping system is shown in figure 4. Two angle plates are used to assemble the hydrofoil on a fixed support: the horizontal sides of the plates are bolted on the hydrofoil through its base and the vertical sides of the plate are bolted on the support. In the hydrodynamic tests, the vertical sides of the plates are directly bolted on the balance (see figure 8). The clamping system are identical for air and hydrodynamic tests. A laser telemeter Acuity AR 500 (precision of $0.01 \mathrm{~mm}$ and acquisition frequency of $100 \mathrm{~Hz}$ ) is used to measure the displacement of the foils. The entire chord is swept by the laser at different sections $\mathrm{S} 2$ and $\mathrm{S} 3$ by the means of a motorized rail when the load is applied on section S1 (figure 4). Two types of loading are performed in these tests:

- Bending loading (figure 4): The mass $M_{1}=2.2 \mathrm{~kg}$ is hang in the middle of the section $S_{1}$ and the laser measures the vertical displacement of the sections $S_{2}$ and $S_{3}$. The results are the bending displacement of the sections $S_{2}$ and $S_{3}$ thus the bending stiffness of the hydrofoils. BTC is also measured, if present. The clamping system was found to be not totally rigid, resulting in discrepancies up to $\pm 0.2^{\circ}$ on the twist angle.

- Torsional loading: A $225 \mathrm{~mm}$ off-center loading is ap- 


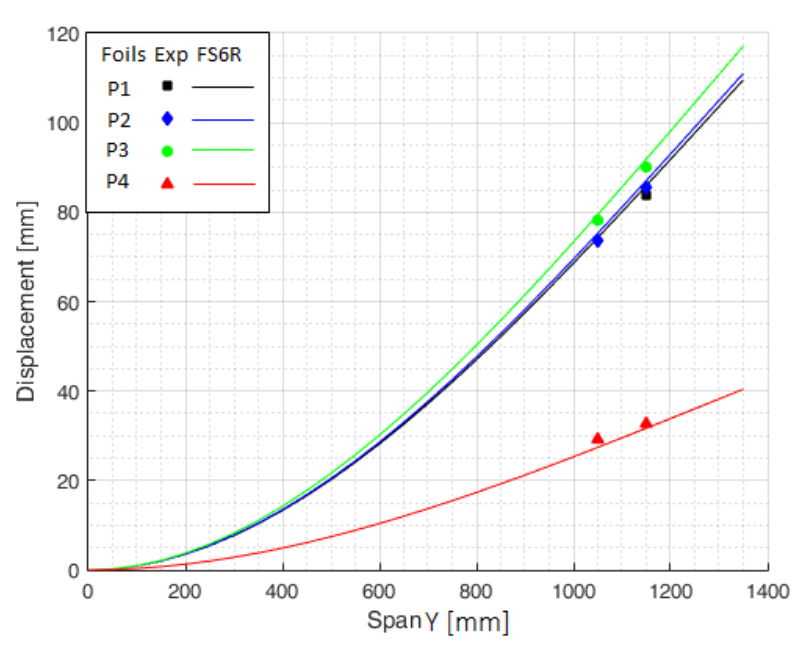

Figure 6: Displacement of the hydrofoils measured in the static tests with the load $M 1=2.2 \mathrm{~kg}$ compared to FS6R results. FS6R is the FSI numerical model described in section 3.1 .

plied on the section $S_{1}$ as shown in figure 5 and the laser measures the vertical displacement of the sections $S_{2}$ and $S_{3}$. To allow only the twist motion, a vertical spike is placed under the hydrofoil to lock the bending motion. The outputs are the twist angles of the sections $S_{2}$ and $S_{3}$ and thus the torsional modulus of the hydrofoils. Four calibrated masses are used leading to the following twisting moments applied on the hydrofoils: $5 N . m, 8.7 N . m, 12.4 N . m$ and $17.5 N . m$.

\subsubsection{Results on the stiffness}

This section presents the results of the bending and torsional stiffness. BTC is discussed in 4.1 in the comparison to numerical simulation. The bending stiffness modulus $E I$ and twisting stiffness modulus $G J$ are calculated using the measured displacement $Y$, the measured twist angle $\theta$ and the geometrical parameters by equation 1 .

$x$ direction is along the chord, $y$ is the vertical direction and $z$ is along the span of the hydrofoil.

$$
E I_{x}=\frac{F \times l_{2}^{2} \times\left(3 l_{1}-l_{2}\right)}{6 Y} \quad G J=\frac{M_{t} \times l_{2}}{\theta}
$$

\section{Bending stiffness}

Figure 6 illustrates the experimental displacements of the four hydrofoils and the bending stiffness calculated by equation 1 are given in table 3 . According to the ply's properties and the layup orientations, $P_{4}$ has the highest bending stiffness, twice as high as the others. The three glass fiber hydrofoil have an equivalent stiffness, $P_{3}$ 's being slightly smaller. The difference between $P_{2}$ and $P_{3}$ is likely due to the manufacturing process. The same observation can be made with the displacements.

\section{Torsional stiffness}

The experimental twist angles recorded at $S_{2}$ for all the layups

\begin{tabular}{|c|c|c|c|c|}
\hline Foil Layup & $P_{1}$ & $P_{2}$ & $P_{3}$ & $P_{4}$ \\
\hline$E I_{x}\left[N . m^{2}\right]$ & 155.6 & 153.8 & 145 & 420.5 \\
\hline
\end{tabular}

Table 3

experimental bending stiffness $E I_{x}$ of the different foils

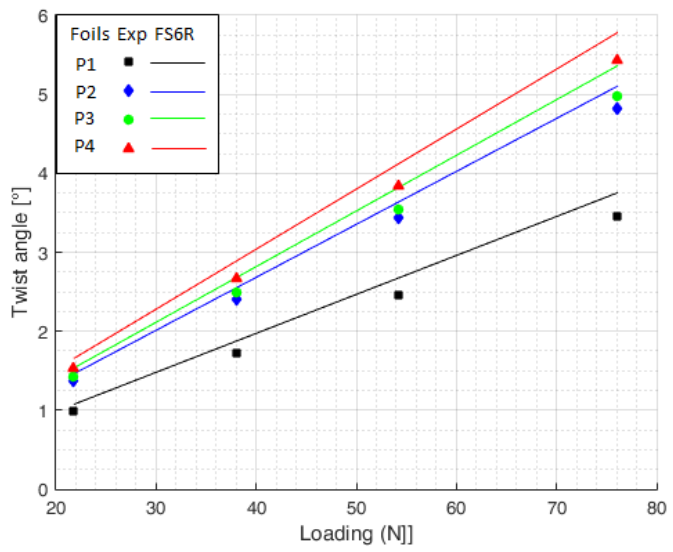

Figure 7: Evolution of twist angle during a static torsion tests for different applied load compared to FS6R simulation (see section 3.1)

\begin{tabular}{|c|c|c|c|c|}
\hline Foil Layup & $P_{1}$ & $P_{2}$ & $P_{3}$ & $P_{4}$ \\
\hline$G J\left[N . m^{2}\right]$ & 290.39 & 216.3 & 203.3 & 186.95 \\
\hline
\end{tabular}

Table 4

Torsional stiffness GJ of the different foils

and the loading cases are presented in figure 7 . The torsional modulus calculated with equation 1 using the measured twist angles are presented in table 4 . The results represent well the expected tendencies due to the different layups.

According to the orientations of $\pm 45^{\circ}$ in the layup of $P_{1}$, its torsional modulus is the highest. The layup $P_{4}$ with only $0^{\circ}$ and $90^{\circ}$ orientations in its layup has the smallest torsional stiffness. As for the bending stiffness, the manufacturing process can be again incriminated for the P2 and P3 differences.

\subsection{Experimental setup of hydrodynamic tests}

Hydrodynamic tests are carried out at IFREMER Lorient 's flume in a working section of $2.5 \mathrm{~m}$ in the flow direction and $1.5 \mathrm{~m}$ depth with a maximum velocity of $1 \mathrm{~m} / \mathrm{s}$ (see Fig. 8). The hydrofoil is clamped to a 6-DOF balance measuring the efforts and moments and the displacements are measured with a laser telemeter through an underwater window (See Fig. 9). The laser and the clamping system are described in section 2.3.1. The precision of the balance is $0.16 \mathrm{~N}$ for the forces and $0.04 N . m$ for the moments. The clamping system of the hydrofoil is attached to the balance and the coordinate system of the force balance is identical to the hydrofoil coordinate system (see figure 8).

The lateral displacements of the hydrofoils are measured at three different heights. At the three different heights, the laser sweeps ten consecutive times the hydrofoil chord wise to average the distance. A calibration process is performed 


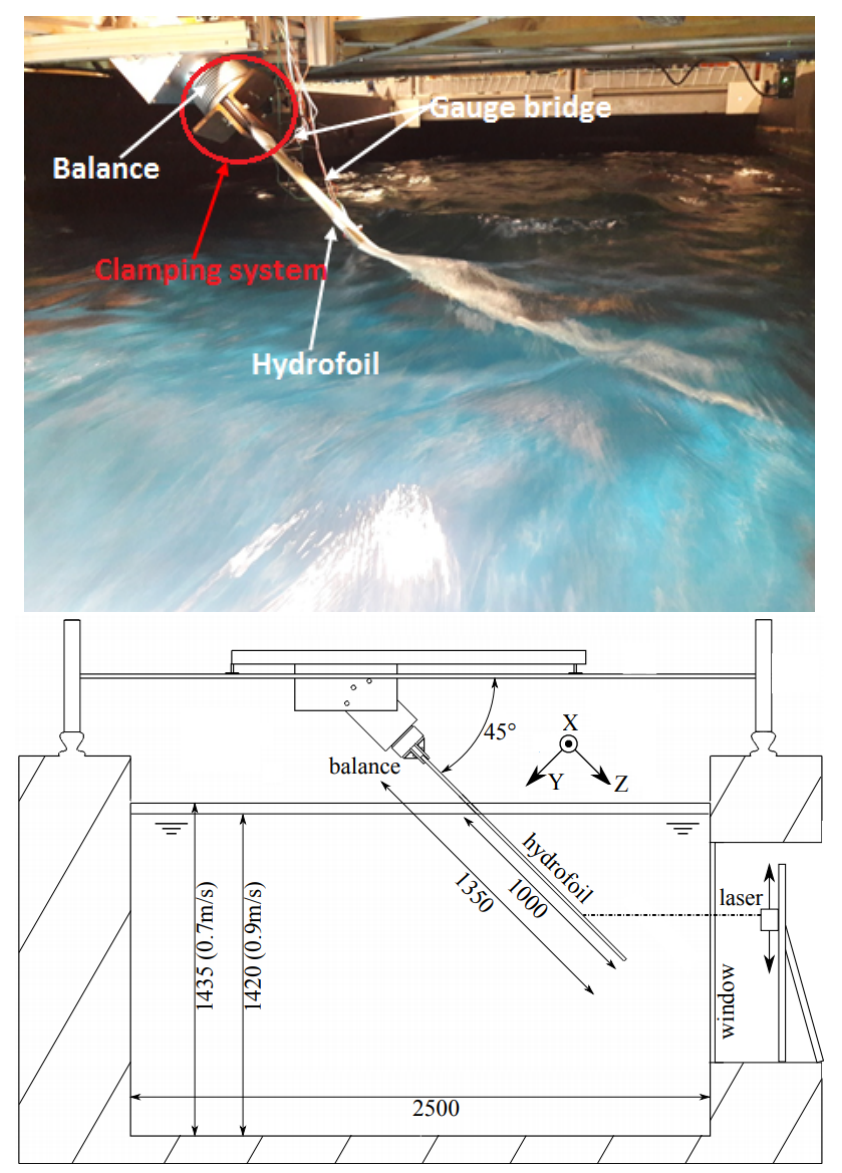

Figure 8: Top: Hydrofoil tested in IFREMER Lorient flume at $0.9 \mathrm{~ms}$ and $\mathrm{AoA}=-9^{\circ}$. The hydrofoil is connected to a $6 \mathrm{DoF}$ balance and pierces the free surface at $45^{\circ}$. Bottom: IFREMER Lorient flume main characteristics and experimental set up with the hydrofoil. Water level changes with flow speed. Dimensions are in $\mathrm{mm}$

prior to the tests to correct the diffraction effect due to the different interfaces that the laser beam encounters. The precision of the measurement is $0.3 \mathrm{~mm}$.

As shown on figures 9 and 8 , the hydrofoils are piercing the free surface with an angle of $45^{\circ}$. The flow is aligned with $\mathrm{X}$-axis. Most of the tests are done with a negative AoA to enhance the laser telemeter measurement and to prevent the tip to get to close to the window of the flume (Fig. 10). The $45^{\circ}$ tilt angle is chosen to simulate the interaction of a foiling yacht hydrofoil with the free surface and to analyze its effects on the hydrodynamic performances. It also has the advantage of maximizing the immersed surface with a low ground effect. Two speeds $0.7 \mathrm{~m} / \mathrm{s}, 0.9 \mathrm{~m} / \mathrm{s}$ and several angles of attack ranging from $\left[-9^{\circ},-3^{\circ}\right]$ are investigated. The free surface height varies with the velocity: $1.435 \mathrm{~m}$ for $0.7 \mathrm{~m} / \mathrm{s}$ and $1.42 \mathrm{~m}$ for $0.9 \mathrm{~m} / \mathrm{s}$.

\section{Numerical Tools}

This section describes the low fidelity and high fidelity tools. The modeling process of the hydrofoils is also pre-

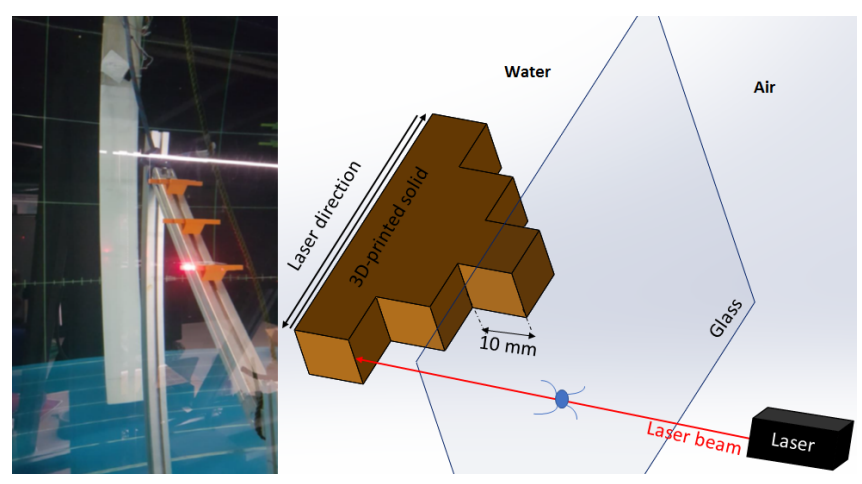

Figure 9: Left: calibration set up for the laser telemeter system in flume. Right: three 3D printed defined geometries (in orange) are placed at the level of the path of the laser.

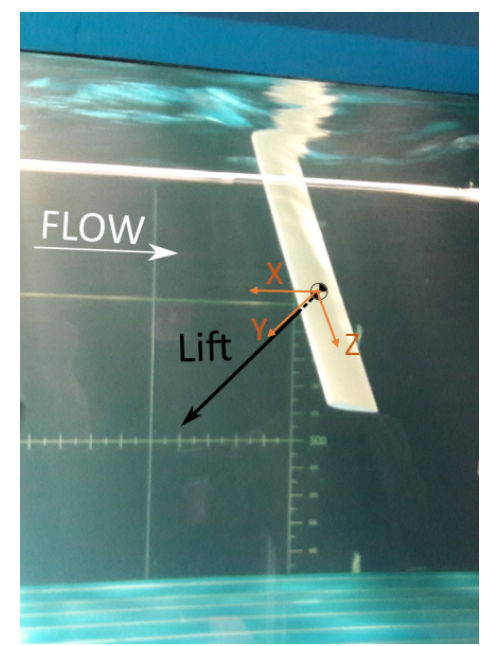

Figure 10: View from the underwater window of a tested hydrofoil mounted at a $45^{\circ}$ angle in the flume. The balance reference frame is represented in orange.

sented for each approach.

\subsection{Low fidelity code: numerical model FS6R}

FS6R is a code dedicated to the preliminary stages of hydrofoils design to model and to analyze the performance with fluid-structure interactions. A vortex lattice method is coupled with an in-house beam theory by finite elements. The organizational chart of the coupling is presented in figure 11 . The process is iterative and works from top to bottom as described below. First, an input file containing the hydrofoil geometry, the flow parameters, the structural materials definition and the configurations to simulate are defined. The first block contains the geometry and mesh from the input file and initiates the coordinate matrices. Then, the hydrodynamic block is activated including two functions: AVL and XFOIL presented in 3.1.1. AVL performs 3D calculations on the whole surface and provides the hydrodynamic coefficients. AVL is a potential code and thus, does not consider the viscosity of the fluid. Therefore a correction is applied using XFOIL software, which performs 2D vis- 
FSI on composite hydrofoils with BTC

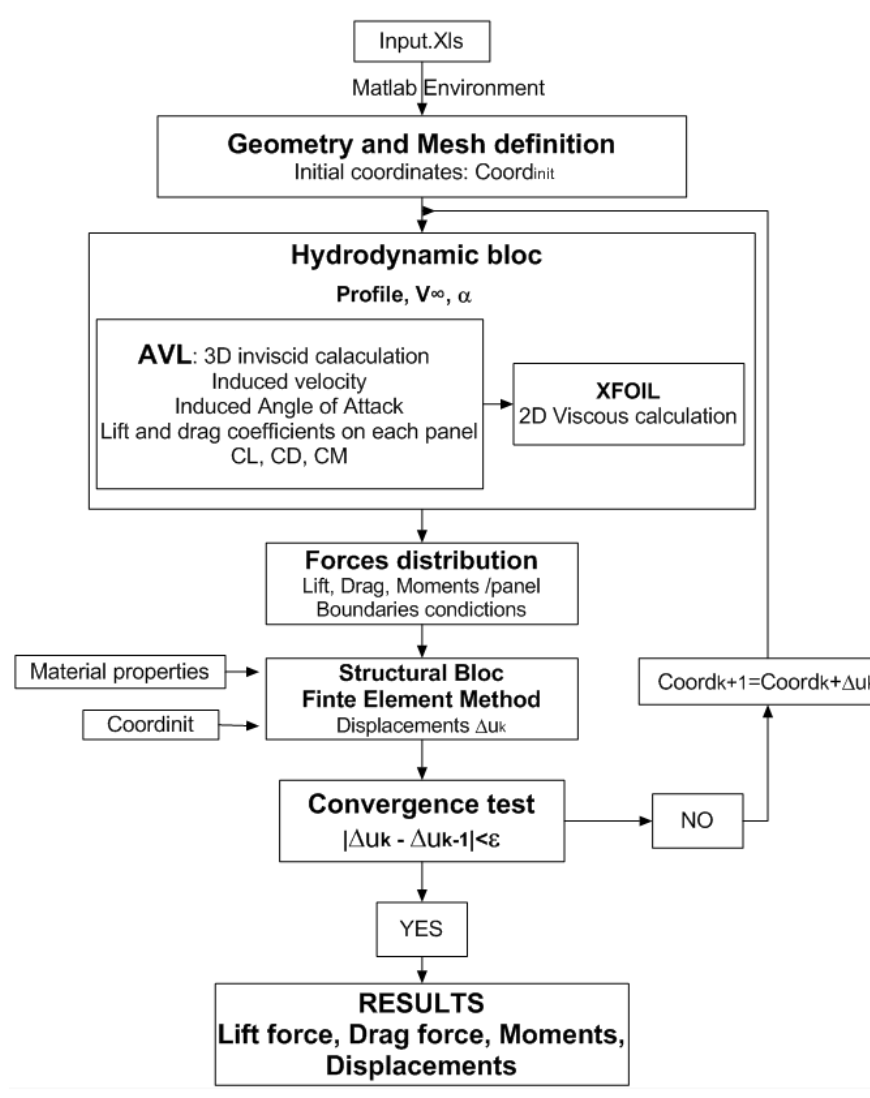

Figure 11: FS6R organizational chart.

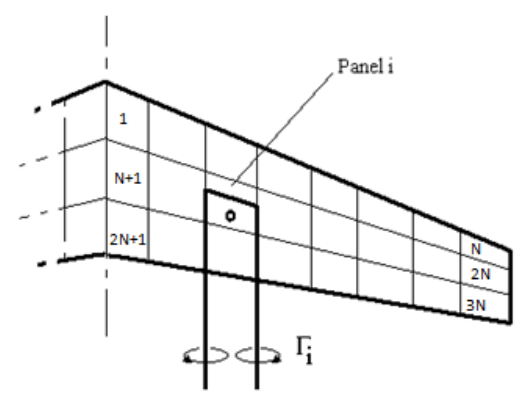

Figure 12: Structure meshing for AVL analysis [6].

cous calculations on each section of the mesh. At the end of this block, the hydrodynamic coefficients distributed over the entire structure are obtained and transmitted to the effort block to calculate the forces distribution. Finite elements calculations is performed in the next bock. It uses the initial mesh, the material definition and the forces distribution to calculate the structural response under these conditions. A convergence test based on the structure's displacement as criteria is performed for a prescribed residue $\epsilon$.

\subsubsection{Vortex Lattice Method and viscous correction}

AVL, Athena Vortex Lattice is a program based on Vortex Lattice Method [12], [6], to analyze arbitrary configurations of rigid aircraft with lifting surfaces and slender body

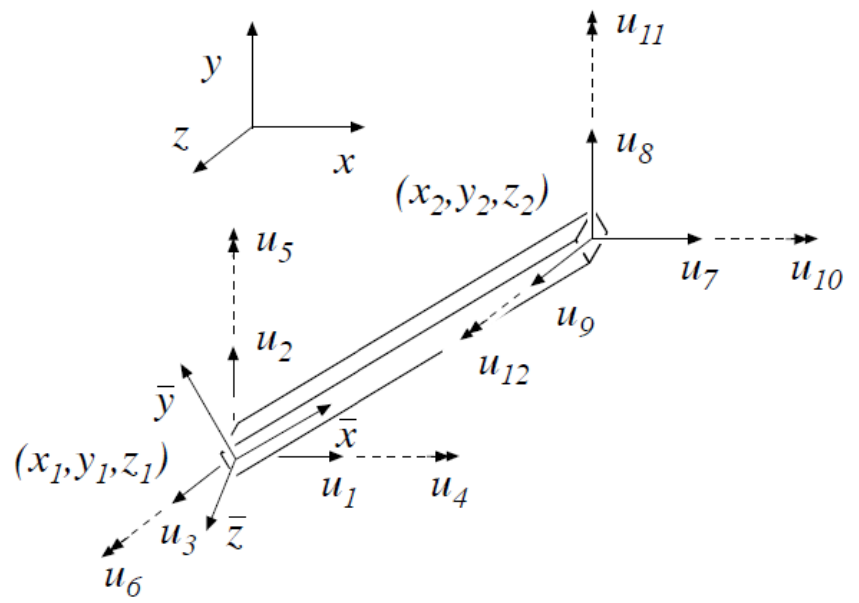

Figure 13: 1 element beam showing the DOF of each node. [3]

model. It is based on the potential flow theory and assumes that the lifting surfaces are thin and modeled with horseshoe vortices distributed along the span and chord while neglecting the influence of viscosity and thickness. Figure 12 shows the hydrofoil discretization into a total of $3 \mathrm{~N}$ panels. Lifting line on each panel is a vortex line normal to the section, located at $1 / 4$ of the panel width in chordwise and to satisfy Thompson law at root and tip, the vortex is aligned with incident flow and extended to infinity. The calculation point in a panel is located at $3 / 4$ in chordwise and $1 / 2$ in spanwise. In agreement with Biot Savart law, a vortex intensity $\Gamma_{i}$ creates an induced velocity normal to the flow velocity. $\Gamma_{i}$ is calculated with geometrical parameters and the induced velocity. This induced velocity modifies the effective angle of attack, creating an induced angle of attack $\alpha_{i}$, which impacts lift coefficient. Lift force per panel is calculated with KuttaJoukowsky theorem. Xfoil [36], [11], performs a viscous correction of AVL calculations. The input are the profile 2D-sections at the calculation point of AVL with the effective angle of attack as incidence. A viscous calculation is performed with Xfoil and the drag force is updated.

\subsubsection{Beam theory by finite elements}

The hydrofoil is modeled as a 3D beam with a linear static structural behavior as shown in figure 13. This beam is prismatic and made of a single isotropic and homogeneous material. The beam is characterized by its neutral axis (along $\bar{x})$ and a straight cross-section within the $(\bar{y}, \bar{z})$ plane. This section stays straight and perpendicular to the neutral axis even after deformation, it is studied using its principal inertial coordinate system $(\mathrm{x}, \mathrm{y}, \mathrm{z})$.

The problem is solved using the displacement finite element method implemented. It has been shown [19] that the problem solution $\left(u_{1}, u_{2}, \ldots u_{11}, u_{12}\right)$ matches the displacement field by minimizing the elastic potential energy $E_{p}$. $E_{p}$ is a function of the strain energy and the work of the external loads (equation 2). The finite element method discretizes the beam 
within $\mathrm{N}$ elements, with two nodes each. A node is characterized by six degrees of freedom, six nodal loads and six shape functions. For an element $e$, the strain energy and the work of external loads are given by equations 3 and $4 .\left\{U^{e}\right\}$ is the nodal displacement vector and $\left[K_{e}\right]$ the element stiffness matrix. $\left\{f_{\text {nod }}^{e}\right\}$ stands for the loads which are directly applied to the nodes.

$$
\begin{aligned}
E_{p} & =E_{\text {def }}-W_{\text {ext }} \\
E_{\text {def }} & =\frac{1}{2}\left\{U^{e}\right\}^{T}\left[K^{e}\right]\left\{U^{e}\right\} \\
W_{\text {ext }} & \left.=\left\{U^{e}\right\}^{T}\left(\left\{f_{d}^{e}\right\}\right)+\left\{f_{\text {nod }}^{e}\right\}\right)
\end{aligned}
$$

Then, $\left[K_{e}\right]$ and $\left\{f_{d}^{e}\right\}$ are assembled for all the elements to obtain the global linear system in equation 5 that we need to solve with boundary conditions.

$$
F=[K] \times U
$$

$\mathrm{K}$ is the assembled stiffness matrix in the global reference $(\mathrm{x}, \mathrm{y}, \mathrm{z}), \mathrm{F}$ represents the forces applied to the structure in the global reference $(\mathrm{x}, \mathrm{y}, \mathrm{z})$ and $\mathrm{U}$, the DOF of the structure in the global reference $(\mathrm{x}, \mathrm{y}, \mathrm{z})$. To solve that equation, many open source tools are available such as FEnics Project, Calculix or CALFEM. The matlab toolbox of CALFEM "Computer Aided Learning (Finite Element Method) [3] is preferred because it is simple to be coupled and it is adaptable for composite hydrofoil simulations. CALFEM is being developed since 70's and the latest update in 2012. Equation 6 express equation 5 for a beam with one element as presented in figure 13. In the force vector and DOF, the bending forces, moment and displacement are highlighted in magenta when the torsional moment and twist are highlighted in salmon. The element stiffness matrix is expressed in the cross section beam reference $((\bar{x}, \bar{y}, \bar{z})$, see figure 13$)$.

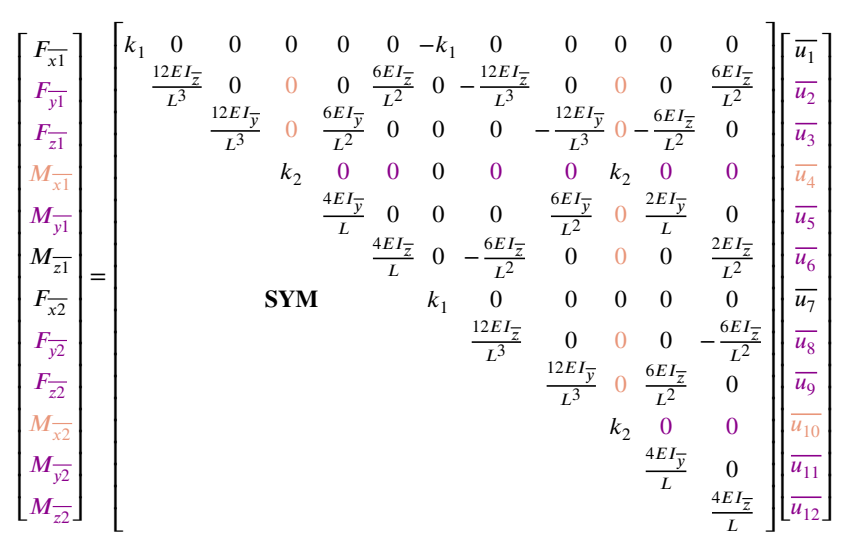

The matrix is expressed using the homogenized crosssection properties: the modulus of elasticity $\mathrm{E}$, the shear modulus $\mathrm{G}$, the cross section area $\mathrm{A}$, the element beam length
$\mathrm{L}$, the moment of inertia with respect to the $\bar{y}$ axis $I_{\bar{y}}$, the moment of inertia with respect to the $\bar{z}$ axis $I_{\bar{z}}$, and St Venant torsional stiffness $K_{v} \cdot k_{1}=\frac{E A}{L}, k_{2}=\frac{G K_{v}}{L}$.

The beam properties are calculated with the variationnal asymptotic method as described in [45]. In that expression of the stiffness matrix, the coupling terms, linking the bending force to a twist motion (highlighted in salmon) and a torsional moment to a bending motion (highlighted in magenta) are usually neglected and set to zero.

\subsubsection{Bend-twist coupling implementation}

Veers and Lobitz [37] introduced a coupling coefficient $\mathrm{g}$ in the stiffness matrix to consider BTC. Equation 7 shows a simplified relation between the torsion and bending moments $M_{t}, M_{b}$ linked to the bending and torsion response of a cross section $k_{b}, k_{t}$, by g. $E I$ is the bending stiffness and $G J$ is the torsion stiffness.

$$
\left[\begin{array}{c}
M_{b} \\
M_{t}
\end{array}\right]=\left[\begin{array}{cc}
E I & -g \\
-g & G J
\end{array}\right]\left[\begin{array}{c}
k_{b} \\
k_{t}
\end{array}\right]
$$

The BTC is created with fibers non aligned with the beam axis [15]. [7] defines the coupling percentage $\alpha$ [\%] (see equation 8 ) as a function of $\mathbf{g}$, the bending stiffness $E I$ and the torsional stiffness $G J$.

$$
\alpha[\%]=\frac{g}{\sqrt{E I G J}}-1<\alpha<1
$$

[35] performed a modal analysis and studied the stability of Bend-Twist Coupled wind turbine blades. From the coupling method of Veers and Lobitz [37], they implemented BTC in the aero-servo-elastic analysis tool HAWCStab2 by modifying the coupling terms in the cross-section stiffness matrix and came out with great results. Therefore, to consider bend-twist coupling in this structural part of FS6R, coupling terms $k_{i j}$ defined in equation 9 are added in the element stiffness matrix (defined in the cross section reference $(\bar{x}, \bar{y}, \bar{z}))$.

$k_{i j}=\sum_{\theta} A_{\theta} \times \alpha_{\theta} \times \sqrt{k_{i i} \times k_{j j}} \quad i=2,3,4,5,6,8,9,11,12, j=4,10(9)$

The expression comes from equation 8 where $k_{i j}$ depends on the coupling percentage $\alpha[\%]$, the torsional stiffness $k_{j j}$ and the bending stiffness $k_{i i} . \theta$ is the orientation of the plies in the layup. A weighting $A_{\theta}$, with $A_{\theta}$ representing the percentage of the ply oriented at $\theta$ degrees in the layup, is added in the coupling terms. The evolution of the coupling percentage with respect to fiber orientation is shown in figure 14 [7] for both glass and carbon.

\subsubsection{Foils modeled with FS6R}

This part describes how the hydrofoils are modeled with FS6R. The geometry is presented as a thin plate separated in two surfaces (one under water and one in air) by a plane located at $\mathrm{z}=$ water free surface height, used to model the free surface. The profile coordinates for each section is determined (here a constant NACA 0015) and used for the AVL 


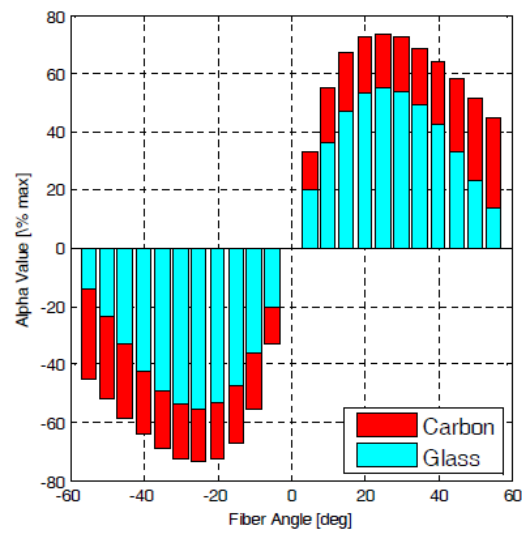

Figure 14: Limit of bend-twist coupling percentage with the plies orientation for glass and carbon fibers. [7]

fluid calculation (see 3.1.1). The structural calculation is performed on the neutral axis of the structure that passes through the twisting centers of the sections. The finite elements and fluid mesh are identical.

\subsection{High fidelity tool: FOAM-ASTER coupling}

To solve the fluid-structure interactions problem in a complete way, a partitioned coupling approach [40] using two open-source codes is implemented: OpenFOAM (Open Field Operation and Manipulation) for the fluid analysis and the thermo-mechanical Code Aster (Analyses des Structures et Thermo-mécanique pour des Études et des Recherches) for the structural analysis.

Figure 15 shows the organizational chart of FOAM-ASTER coupling based on a iterative process working from top to bottom. The method is an explicit unpartionned weak coupling based on a stationary approach. OpenFOAM is the first block using Gauss-Seidel iterations with as inputs: the geometry file $*$.st $l$ and the running case parameters (flow velocity $V_{\infty}$, angle of attack $\alpha$, turbulence model and the transport properties $(\rho, v, \ldots))$. The mesh is performed with CfMesh, a mesh generator tool compatible with OpenFOAM. The fluid mesh coordinates are stored in $\left[C_{f}\right]$. Then, the fluid simulation is performed giving as outputs: the mesh coordinates $\left[C_{f}\right]$ and the load distribution $\left[F_{f}\right]$. The structural mesh is performed by the CAD tool Salome on the initial geometry. The second block of the coupling is Code_Aster which takes as input the structural mesh, the material properties, the boundary conditions, the fluid mesh coordinates and the forces distribution on the structure from the fluid block. The loads from the fluid mesh are translated on the structural mesh according to a python interpolation "griddata" [34]. It takes as inputs the loads on the fluid mesh, $\left[F_{f}\right]$, the coordinate of the fluid mesh $\left[C_{f}\right]$, the coordinate of the structural mesh $\left[C_{S}\right]$ and a method type to perform the interpolation $\left[F_{S}\right]$ of the loads on the structural mesh:

$$
F_{S}=\operatorname{griddata}\left(\left[C_{f}\right],\left[F_{f}\right],\left[C_{S}\right], \text { type }\right)
$$

The mechanical analysis is performed with the fluid load-

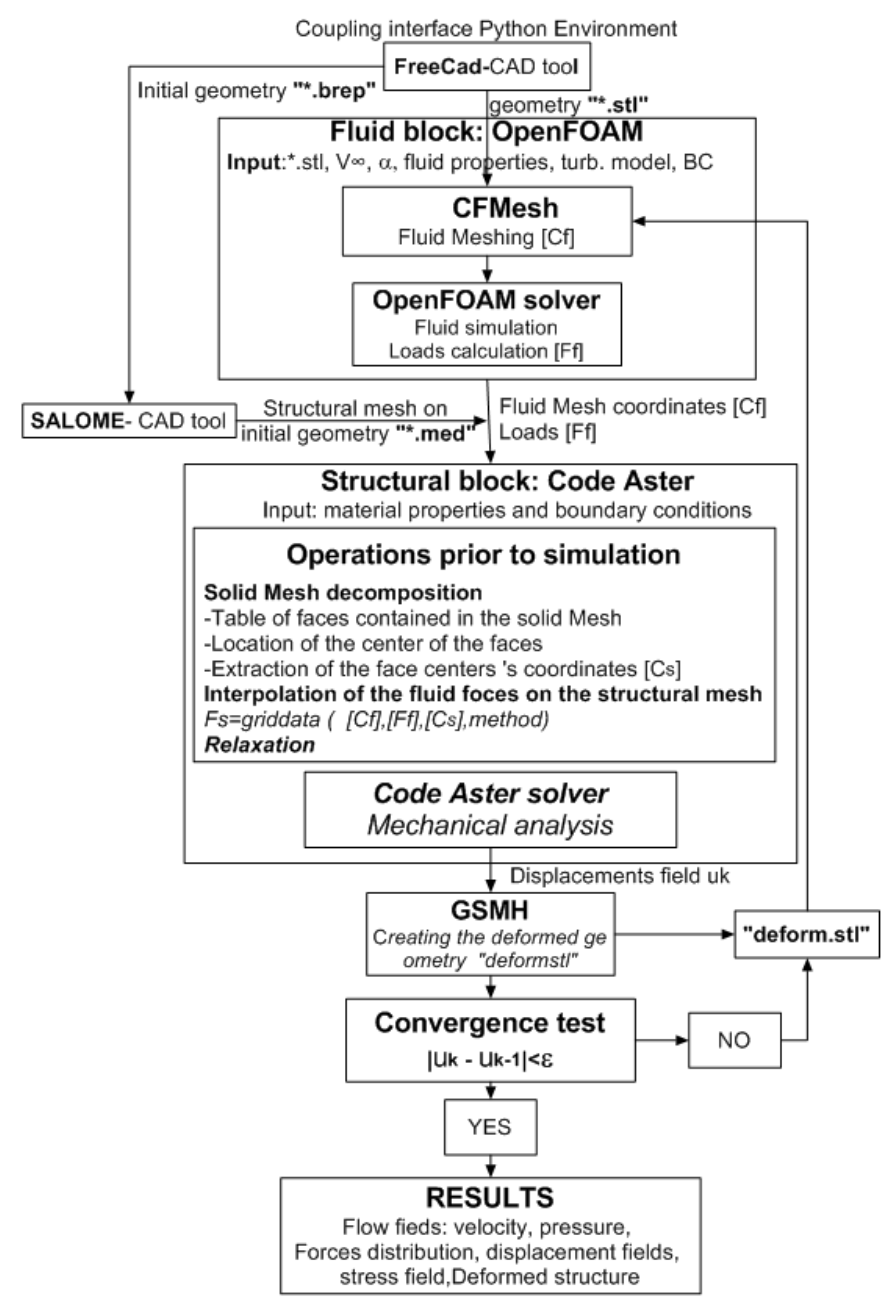

Figure 15: organizational chart of FOAM-ASTER coupling

ing giving as outputs: the displacement field and the stress field of the structure. The deformed geometry "deform.stl" is built from the structural displacement with the help of the mesh generator tool Gmsh. A convergence test is performed based on a displacement criteria. If the convergence is not reached, a new iteration starts with "CfMesh" using "deform.stl" as input.

The outputs of the FOAM-ASTER coupling are: The flow field (velocity, pressure, ...), the loads distribution on the structure, the displacements, the stress field and the deformed structure.

\subsubsection{Fluid code OpenFOAM}

OpenFOAM is an open code based on a development framework principle for CFD solvers (Computational Fluid Dynamics).

The OpenFOAM solver InterFoam [9] is chosen to model the flow around the hydrofoils piercing the free surface. It is a multiphase flow solver for 2 incompressible, isothermal immiscible fluids using a VOF phase-fraction [22] approach, to capture the interface.

Shaoshi [8] evaluate OpenFOAM for use in the field of Ocean 
engineering. Simulations of the flow field around a fullscale Tension-Leg Platform (TLP) in steady current at high Reynolds number were performed and OpenFOAMs ability to accurately predict the unsteady hydrodynamic loads was assess. The results validate suitability of this open-source tool, when used with the appropriate turbulence closure.

[42] describes the principles behind the computationnal opproach of OpenFOAM and the OpenFOAM User Guide [1] presents the solver features and how to execute them in a complete way.

\subsubsection{Structural code Aster}

The solvers of the structural code Aster [10]are based on the theory of the mechanics of continuous media and uses the finite element method to solve different types of mechanical, thermal, acoustic, seismic and other problems. 1D, 2D and 3D models such as beam, shell and 3D solid are performed with a wide range of finite elements types.Code_Aster allows various type of material behaviors such as elastic, orthotropic materials (polymers, metals and composites, etc). This code has an important level of validation similar to the commercial structural code Abaqus.[2] presents a simple use of Code aster in a wide range of applications.

\subsubsection{Modeling of the hydrofoils with FOAM-ASTER}

This part presents the modeling of the hydrofoil for OpenFOAM and code ASTER calculation.

\section{Fluid model}

Figure 16 shows the fluid domain meshed and a zoom on the profile showing the boundary layer around the foil. The domain size is $5 \times 2.5 \times 2.5 \mathrm{~m}^{3}$ for $1.5 \times 10^{6}$ hexahedra cells of unstructured mesh; the hydrofoil is placed in the same configuration than the experiment figure 8. The fluid simulation carried out with the OpenFOAM solver interFoam uses the $K$-OmegaSST for the turbulence model. A non-slip wall boundary condition is imposed on the hydrofoil with a wall function movingWallVelocity. The time interval is chosen to have an initial Courant number equals to 1 .

\section{Solid modeling of the foils}

The solid structure is modeled to consider the composite material and the sandwich section made of a skin and a web. The structural mesh is presented in figure 16. The structure is a hollow hydrofoil composed of a laminated skin defined by its layup (table 1) and a web made of an isotropic material. The skin and the web are modeled with shell elements DKT type, based on the Love-Kirchoff bending model and include triangular and quadrangular finite elements. This finite element plate modeling is intended for small deformation calculations and is considered to be among the most accurate for displacement calculations [24].

The boundary conditions simulates the clamping system as a cantilever free condition. The pressure loads and viscous loads are applied on each center node of the structural mesh as described in 3.2. The outputs of the simulation are the displacements and stress fields.

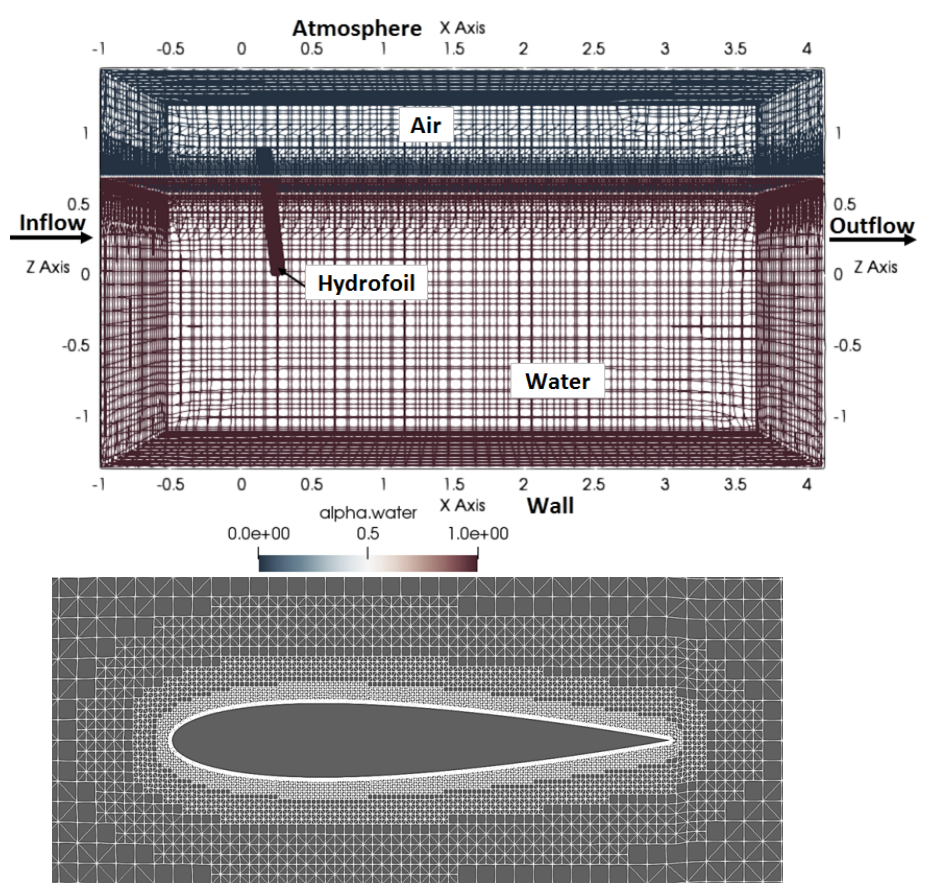

Figure 16: Mesh of the fluid domain and zoom on the hydrofoil section mesh.

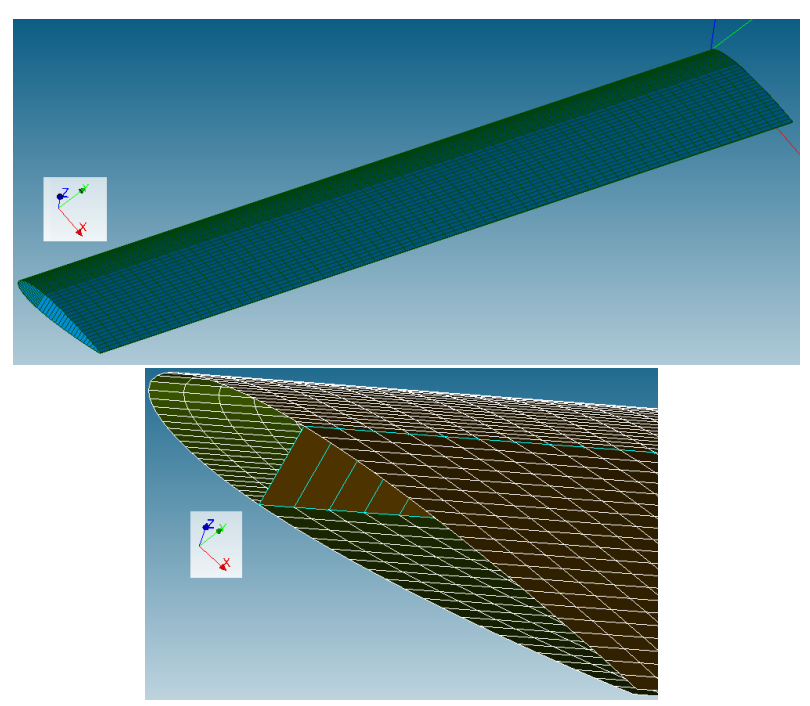

Figure 17: Structural model composed of a skin and a web modelled with shell elements.

\section{Results and discussion}

This section describes the numerical and experimental comparison on BTC for the static tests in air and for the hydrodynamic tests in the flume.

\subsection{Coupled bending and torsional effect}

This section presents the results of the BTC investigation performed during the static bending tests.

Note: The twist angle is positive for a clockwise rotation and negative for counter clockwise rotation.

Figure 18 shows the bend-twist angle measured at $S_{2}$ and $S_{3}$ for all the layup. The experimental measurements are com- 


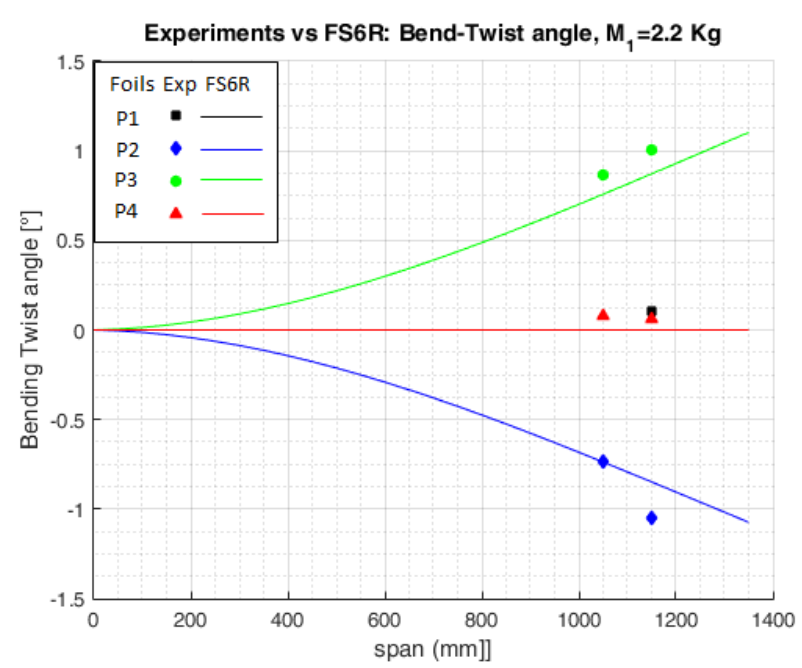

Figure 18: Twist angle due to BTC: static experiments in air VS FS6R numerical approach.

pared to the results of the FS6R approach considering the bend-twist coupling terms in the stiffness matrix. The results computed with FS6R method are very close but lower than the experimental values. The relative discrepancies are less than $9 \%$ on the twist calculation. The underestimation of the torsional modulus is directly linked to the coupling terms between the modulus $E I$ and $G J$ showed in matrix 6 that was determined by a pure torsion test in static. The measured angle were small and may induced an error in the coefficient calculation.

FS6R computes a zero twist angle for $P_{1}$ and $P_{4}$, accordingly to the balanced layup of both composites. A small experimental twist of $0.1^{\circ}$ were recorded for $P_{1}$ and $P_{4}$, which is the order of magnitude of the measurement precision. The manufacturing process could be, as well, a contributing factor. As expected, a twist angle coupled to the bending for the layup $P_{2}$ and $P_{3}$. The twist angle reaches $1^{\circ}$, rather positive or negative, depending on the orientation of the $45^{\circ}$ plies. FS6R simulates well the observed tendencies.

\subsection{Hydrodynamic tests}

This section presents the numerical and experimental comparison of loads and displacement of the hydrofoils in flume. Both numerical coupling, FS6R and FOAM-ASTER are compared. Experiments were initially design to reproduce the condition encounter by high performance sailing yacht where the interaction with the free surface is significant. Due to low testing speeds, no curvature and high aspect ratio, this interaction is very limited, minimizing the advantage of a two phase simulation as done in FOAM-ASTER.

\subsubsection{Foil displacements}

The hydrofoils are subject to small amplitude oscillation when in the flume with flow. The vibration are mainly due to the free surface variation, difficult to completely smooth at the tested speeds. The displacement are average on $10 \mathrm{~s}$ to fit the quasi-steady numerical approach. The hydrofoil displacements are measured at three different span positions with the laser telemeter. The displacements for $-5^{\circ}$ and $-7^{\circ}$ at $0.9 \mathrm{~m} / \mathrm{s}$ are represented in figure 19 . The experimental values are compared to FS6R simulations with BTC for all cases. The hydrofoils have a different behavior in water than the one observed in air (Fig. 6). $P_{3}$, which experienced in air the more displacement, due to module of elasticity weakness, has now less deformation than $P_{1}$ and $P_{2}$, respectively. $P_{2}$ which has the same behavior than $P_{1}$ in air has now more deformation.

The results can be explained by BTC due to the $45^{\circ}$ plies in the layup. The $-45^{\circ}$ orientation of the plies in the $P_{2}$ composite structure leads to a negative twist of the structure when bending, with the direct consequence of loading the tip and increasing the displacement (negative incidence are investigated here). The opposite phenomenon is observe on $P_{3}$ where the composite structure contains $+45^{\circ}$ plies with the tendency of unloading the tip. For $\alpha=-7^{\circ}$, the displacement at the tip rises up to $77 \mathrm{~mm}$ (5.8\% of span) for $P_{2}$ glass fiber and only $68 \mathrm{~mm}(5.01 \%)$ for the $P_{3}$ when $P_{3}$ and $P_{2}$ hydrofoils layup only differs by the orientation of $45^{\circ}$ plies.

As expected in the design process and in agreement with its highest bending stiffness (see table 3), the carbon hydrofoil $P_{4}$ experiences the lowest displacement with $40 \mathrm{~mm}$ at the tip (3\% of its span length).

These measurements highlight different behaviors that are linked both to the composite layup and the materials of the tested foil. First, the carbon foil, with the same number of plies is significantly more rigid than the glass fiber foils. The second behavior is directly linked to the subject of this paper, the bend-twist coupling. As illustrated in the figure 14, the coupling coefficient $g$ in the mechanical matrix, described in section 3.1.3, differentiates significantly the simulated displacement of $P_{2}$ and $P_{3}$ and leads to a good fit of the experimental results.

The simulations performed by FS6R fit well the observation showing the ability of the code to compute the different layup behaviors and the bend-twist coupling.

\subsubsection{Effects on hydrodynamic loads}

The potential flow theory and Xfoil calculations give good results for drag coefficient but is limited to a $2 \mathrm{D}$ approach. In the studied case, the representation in FS6R of the free surface by a mirror plate improves the lift results but can't simulate the wave drag. the hydrofoils piercing the free surface induce waves in the flow with a consequence of an increase in the drag force. The calculated drag tendencies of the potential flow coupling FS6R are good but the order of magnitude are not good enough to be presented here.

The Lift force recorded during the experiments and the simulations performed with FS6R for all the incidences at a flow speed $U_{\infty}=0.9 \mathrm{~m} / \mathrm{s}$ are presented in figure 20 . The vertical bars are the standard deviation of the measurements. Lift magnitude and its projections on Y-axis $\left(F_{y}\right)$ and Z-axis $\left(F_{z}\right)$ are also illustrated for the 4 hydrofoils in the figure. The lift is defined as magnitude of the measured $F_{y}$ and $F_{z}$ components. Experimental and FS6R results have the same trend 

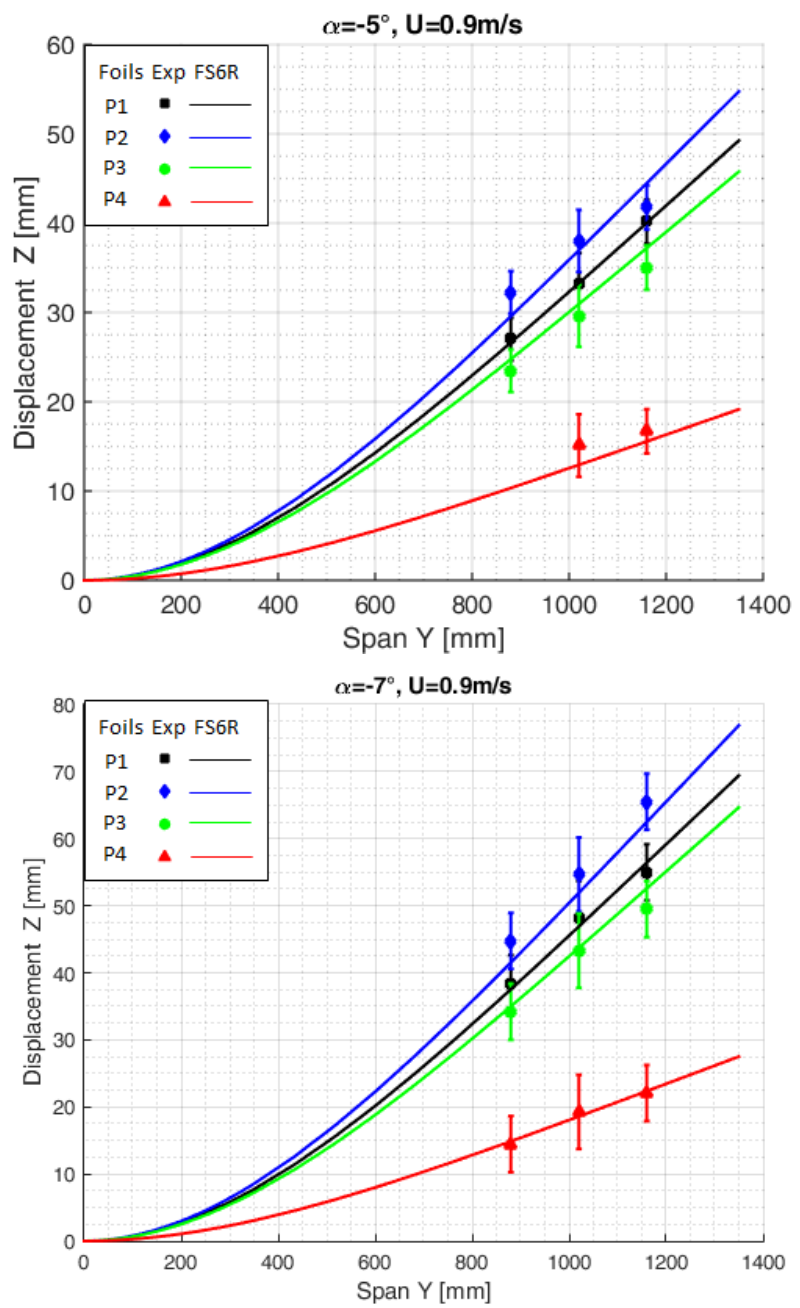

Figure 19: Experimental and numerical comparison based on the coupled calculation FS6R for the 4 hydrofoils at flow speed $U=0.9 \mathrm{~m} / \mathrm{s}$ for different AoA. Displacements are measured at 3 spans location with a laser telemeter. Top: Displacement at $\alpha=-5^{\circ}$. Bottom: Displacement at $\alpha=-7^{\circ}$. The vertical bars are the standard deviation of the measurements.

and the values fits well excepts for $Z$ projection of the lift force. The $\mathrm{Z}$ value is very small and does not affect the lift force nor the moment leading to a good agreement of both approaches. The maximum discrepancy observed is around $5 \%$ on the lift force for $P_{3}$, with the simulation overestimating the experiments (same observation on the displacements).

The lift force clearly exhibits the different behaviors of the layups: $P_{1}$ and $P_{4}$ overlap with the rigid case as expected, highlighting no bend-twist effect. $P 3$ is smaller than the rigid case and $P_{2}$ is higher. The results are in consistent with the described BTC: $P_{2}$ with the negative twist is the most loaded when $P_{3}$ with the positive twist is the less loaded.

We also observe that when $P_{1}$ and $P_{4}$ lift magnitude are the same, their projections $F_{y}$ and $F_{z}$ are different due to their different bending deformation. $F_{z}$ from FS6R computations has the same trend as the displacements, the force is transferred to the span wise direction due to the hydrofoil deflection,
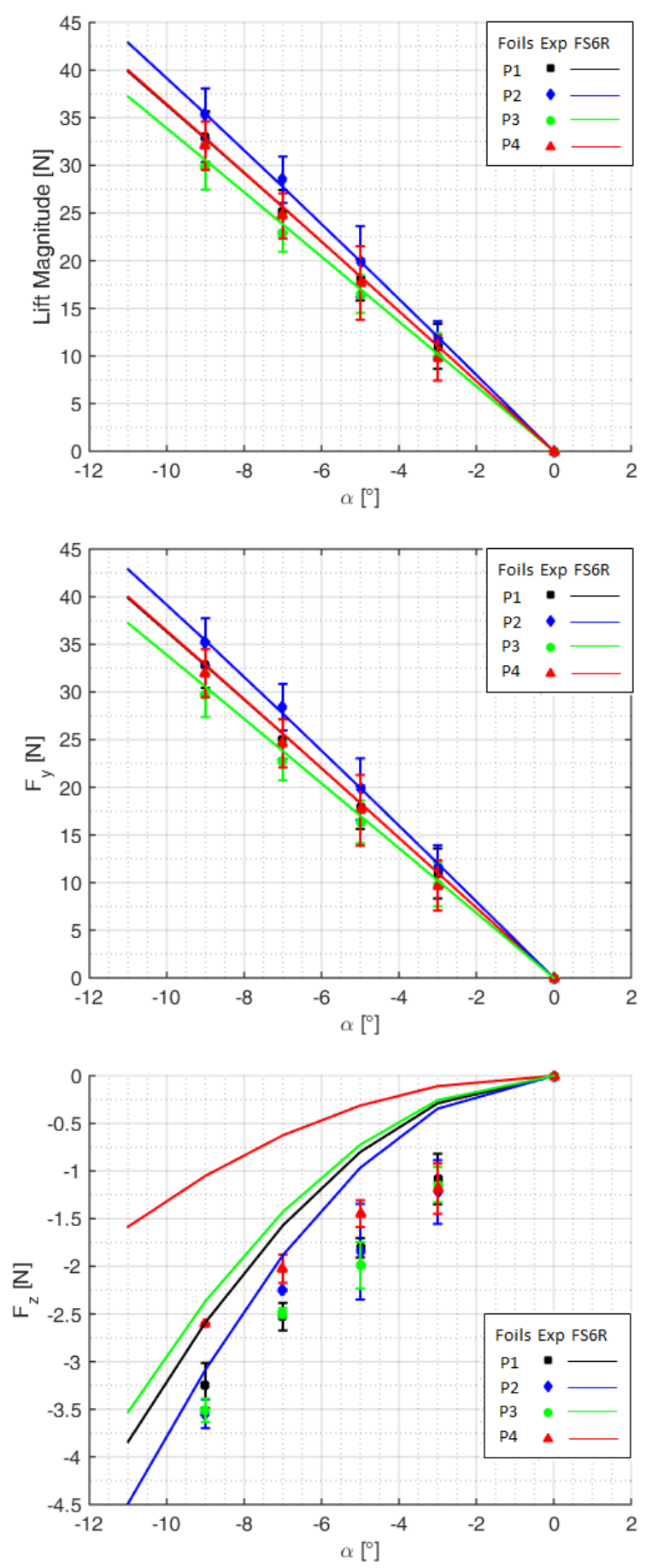

Figure 20: Experimental and numerical comparison based on the coupled calculation FS6R for the 4 hydrofoils at flow speed $U=0.9 \mathrm{~m} / \mathrm{s}$ for different AoA. The forces measured with a $6 \mathrm{DoF}$ balance connected at the root of the hydrofoils.

modifying the hydrodynamic behavior. We observe that $F_{y}$ match very well in both numerical approaches and experimental values are slightly higher.

As a conclusion, FS6R is able to estimate the impact of bendtwist coupling on lift force distribution with good accuracy. The FSI induces significant deformation, impacting the force 

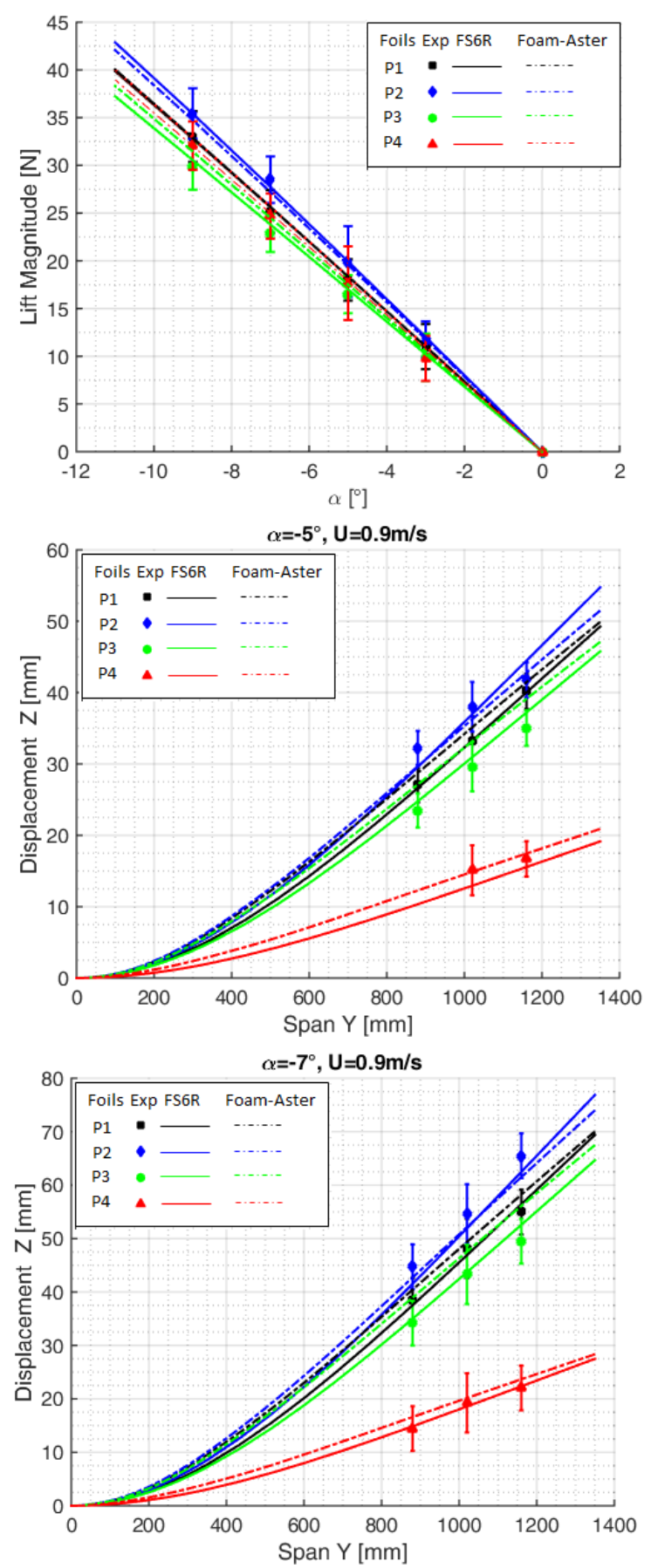

Figure 21: Experimental and numerical comparison based on the 2 coupled calculations FS6R and Foam-Aster for the 4 hydrofoils at flow speed $U=0.9 \mathrm{~m} / \mathrm{s}$ and AoA $\alpha=-5^{\circ}$. Top: Lift magnitude. Bottom hydrofoil displacement along the span.

distribution and eventually the balance of the system, i.e. the sailing yacht.

\subsubsection{FOAM-ASTER Results}

The coupling OpenFOAM-Aster is compared to the experiments and the numerical approach FS6R.

Figure 21 compares the results of the three approaches.
The vertical bars are the standard deviation of the measurements. The lift for different angles of attack is presented in the first graph where the displacements computed for $\alpha=-5^{\circ}$ is in the second graph. The three approaches give the same trend. FOAM-ASTER coupling gives good results on the hydrodynamic forces and the displacement simulations. The results of FOAM-ASTER on the lift force are slightly lower than FS6R and the difference with the experiments is less than 5\%. Looking at the displacements, the results fit well with experiments for $P_{4}$ and a difference around 5\% is observed with $P_{1}$. This coupling method simulates the bendtwist coupling for $P_{2}$ and $P_{3}$ but the twist angle is underestimated. The over estimation of the torsional stiffness by FOAM-ASTER minimise the BTC and impacts the calculated displacement. FOAM-ASTER results are then the lowest in the three approaches, for both $P_{2}$ and $P_{3}$. The difference with the experiments are less than $8 \%$.

The fact that the 'low fidelity' tool FS6R performed better than the more complex coupling FOAM-ASTER is mainly due to the studied case. Indeed, the high aspect ratio and the simple composite sandwich structure constant along the span fits the hypothesis of the beam theory and the potential theory. Moreover, the free surface deformation, visible experimentally where the hydrofoil is piercing the surface, is of second order due to the low AoA and flow speed. Comparison of the two numerical approaches may differ with a more complex composite structure and flow. Finally, the structure properties used in $F S 6 R$ are extracted from the static calibration of the composite structure when FOAM-ASTER computes the properties from the layup and the mechanical properties of the components. It highlights the great results of FOAM-ASTER coupling.

\section{Conclusion}

This work presented an experimental and numerical study of the impact of the composite layup on the hydrodynamic performances of a surface piercing hydrofoil. Two different coupled numerical approaches are described to simulate the FSI of composite hydrofoils subject to hydrodynamic loading. The first tool, FS6R, dedicated to the first stage of the design, is a coupling between the potential flow theory and the beam theory. The second tool, FOAM-ASTER, is a high fidelity approach coupling an OpenFOAM VoF method with the structural code Aster.

Four hydrofoils are built from the same mold with different materials and layups. Mechanical characterisation of the four hydrofoil have highlighted different behavior due to the designed structural differences. Bending-Twisting Coupling [BTC] has been observed for the hydrofoils having $45^{\circ}$ plies. Numerical approaches are able to simulate these differences with the good order of magnitude of the twist angles and the bending displacements.

Hydrodynamic experiments are performed in a flume where the four hydrofoil are cantilevered and surface piercing the free surface at $45^{\circ}$ for different speed and angle of attack. The previously highlighted BTC has here an important effect due 
to the amplification action of FSI. The hydrofoil $P_{2}$ where the BTC tends to increase the AoA (positive BTC) at the tip, experiences more lift and thus more deformation than the 2 hydrofoils $P_{1}$ and $P_{4}$ where the layup cancels the BTC. The opposite observation can be made on the hydrofoil $\mathrm{P} 3$ where the $45^{\circ}$ plies of the layup are in the other direction leading to a negative BTC.

Numerical and experimental comparison gives good results for both numerical coupling, showing the ability of the tools to simulate the hydro-elastic response of a composite hydrofoil with BTC. FS6R, which can be consider has a low fidelity tool, gives very good results in this academic configuration but could show more discrepancies for more complex and thus realistic composite structure as well as for stronger interaction with the free surface.

\section{CRediT authorship contribution statement}

TEMTCHING TEMOU Vanilla: Experimental study, numerical development, data processing, writing. AUGIER Benoit: Supervision of the project, Experimental study, data processing, writing. PAILlARD Benoit: Numerical development, data processing, writing.

\section{Acknowledgements}

Authors would like to thank Benoit Vincent and the staff of IFREMER Lorient's flume for their support, Nicolas Dumergue for his contribution on composite modelling, Yohann Quistrebret for his help in the manufacturing of the hydrofoils and, Thomas Dalmas for his collaboration during the experiments.

\section{References}

[1] OpenFOAM user Guide, version6.

[2] J.-P. Aubry. Beginning with code Aster: A practical introduction to finite element method using code Aster and Gmsh, 2013-2019.

[3] P-E. Austrell, O. Dahlblom, A. Olsson Lindemann, K-G. Olsson, K. Persson, H. Petersson, M. Istinmaa, G. Sandberg, and P-A. Wernberg. CALFEM manual, a finite element toolbox version 3.4. Copyright 1992-2004 by the Division of Structural Mechanics at Lund University. All rights reserved, 1992-2004.

[4] R. Balze, N. Bigi, K. Roncin, and J.-B. Leroux. Racing. Innovsail International Conference, Lorient, France, pages 51-58, 2017.

[5] C. L. Bottasso, F. Campagnolo, C. Croce, and Tibaldi. Optimizationbased study of bend-twist coupled rotor blades for passive and integrated passive/active load alleviation. In Wind Energy, 16, 1149-1166, https://doi.org/10.1002/we.1543, 2013.

[6] K. Budziak and D. Scholz. Aerodynamic Analysis with Athena Vortex Lattice (AVL). Master project, MSME, Department of Automotive and Aeronautical Engineering Hamburg University of Applied sciences, 2015.

[7] M. Capellaro. Design challenges for bend twist coupled blades for wind turbines and application to standard blades. In Proceedings of Sandia Wind Turbine Blade Workshop, 2012.

[8] S. Dai, A. Y. Bassam, and S. Liping. Openfoam predictions of hydrodynamics loads on full-scale tlp. Ocean Engineering, 102:162 - 173, 2015.

[9] S. M. Damián. Description and utilization of interFoam multiphase solver, 2012.
[10] Electricité de France. Finite element code_aster, analysis of structures and thermomechanics for studies and research. Open source on www.code-aster.org, 1989-2017.

[11] M. Drela. Xfoil: An analysis and design system for low reynolds number airfoils. Springer Berlin Heidelberg, 54:112, 1989.

[12] M. Drela and H. Youngren. AVL:Extended Vortex Lattice Model-3.36 User Primer, last update 12 Feb 17.

[13] A. Ducoin, J.-A. Astolfi, F. Deniset, and J.-F. Sigrist. Computational and experimental investigation of flow over a transient pitching hydofoil. European Journal of Mechanics B/ Fluids, 28:728 - 743, 2009.

[14] A. Ducoin, J. A. Astolfi, and J.-F. Sigrist. An experimental analysis of fluid structure interaction on a flexible hydrofoil in various flow regimes including cavitating flow. European Journal of MechanicsB/Fluids, 36:63-74, 2012.

[15] V. Fedorov. Bend twist coupling effect in wind turbine blades. Phd thesis, Technical University of Denmark, 2012.

[16] F. Gaugain. Analyse expérimentale et simulation numérique de l'interaction fluide-structure d'un hydrofoil élastique en écoulement subcavitant et cavitant. Thèse de Doctorat, Ecole Nationale Supérieure d'Arts et Métiers - Institut de Recherche de l'Ecole Navale, 2013.

[17] L. M. Giovannetti. Fluid structure interaction testing, modeling and development of Passive Adaptive Composite foils. Ph.d thesis, University of Southampton, 2017.

[18] L. M. Giovannetti, J. Banks, M. Ledri, S.R. Turnock, and S.W. Boyd. Toward the development of a hydrofoil tailored to passively reduce its lift response to fluid load. Ocean Engineering, 167:1-10, 2018.

[19] D.H. Hodges and H. Dewey. Nonlinear Composite Beam Theory, . 781-56347-697-6. American Institute of Aeronautics and Astronautics, $1,2006$.

[20] S. Hoerner, T. Maître, C. Bonamy, and D. Thévenin. An experimental and numerical study of fsi applied to sail yacht flexible hydrofoil with large deformations. In Proceedings of 9th International Symposium on Fluid-Structure Interactions Flow-Sound Interactions FlowInduced Vibration and Noise (Toronto Canada), 2018.

[21] B. Horel and M. Durand. Application of a system-based modelling and simplified-fsi to a foiling open 60 monohull. the 23th Chesapeak Sailing Yacht Conference, Annapolis, USA, 23:1-10, 2019.

[22] H. Jasak. Error Analysis and Estimation for the Finite Volume Method with Applications to Fluid Flows. Ph.d thesis, Imperial College of Science, Technology and Medicine, London, 1996.

[23] H. J. T. Kooijman. Bending-torsion coupling of a wind turbine rotor blade. In Smart Rotor Project, 1996.

[24] A. D. Kudawoo. Eléments de plaque : modélisations dkt, dst, dktg et q4g. https://www.codeaster.org/V2/doc/default/fr/manr/r3/r3.07.03.pdf, 2018.

[25] Y. Liao, N. Garg, J.R. Martins, and Y.L. Young. Viscous fluid-structure interaction response of composite hydrofoils. Composite Structures, 212:571 - 585, 2019.

[26] Y. Liao, S. He, J. Martins, and Y.L. Young. Hydrostructural optimization of generic composite hydrofoils. In AIAA Scitech 2020 Forum, page $0164,2020$.

[27] Y. Liao, J.R. Martins, and Y.L. Young. Sweep and anisotropy effects on the viscous hydroelastic response of composite hydrofoils. Composite Structures, 230:111471, 2019.

[28] D. Lobitz and P. Veers. Load mitigation with bending/twist-coupled blades on rotors using modern control strategies. Wind Energy, 6:105 - 117, 042003.

[29] D. Lobitz, P. Veers, G. Eisler, D. Laino, P. L. Migloiore, and B. Gunjit. The use of twist-coupled blades to enhance the performance of horizontal axis wind turbines. 012001.

[30] T. H. Manudha, K.L. Aaron, and B. G. Prusty. Design of shapeadaptive wind turbine blades using differential stiffness bend-twist coupling. Ocean Engineering, 95:157 - 165, 2015.

[31] L. Pernod, A. Ducoin, H. L Sourne, J.-A. Astolfi, and P. Casari. Experimental and numerical investigation of the fluid-structure interaction on a flexible composite hydrofoil under viscous flows. Ocean Engineering, 194:106647, 2019. 
[32] A. W. Phillips, R. Cairns, C. Davis, P. Norman, P.A. Brandner, B.W. Pearce, and Y.L. Young. Effect of material design parameters on the forced vibration response of composite hydrofoils in air and in water. In Fifth International Symposium on Marine Propulsors, pages 813822, 2017.

[33] W. Raither, A. Bergamini, and P. Ermanni. Profile beams with adaptive bending-twist coupling by adjustable shear centre location. Journal of Intelligent Material Systems and Structures, 24(3):334-346, 2013.

[34] Scipy.org. SciPy V1.3.2 Reference Guide: scipy.interpolate.griddata, 9 Nov. 2019.

[35] A. R. Stäblein, H. M. Hartvig, and D. R. Verelst. Modal Properties and Stability of Bend-Twist Coupled Wind Turbine Blades. Wind Energy Science, 2017.

[36] V. Storch and J. Nozicka. On viscous-inviscid interaction for boundary layer calculation using two-equation integral method. In Studentska tvurci cinnost 2015. CTU in Prague, Faculty of Mechanical Engineering, 2015.

[37] P. Veers, D. W. Lobitz, and B. Gunjit. Aeroelastic tailoring in windturbine blade application. In Windpower '98, American Wind Energy Association Meeting and Exhibition,Bakersfield, California, April 28 - May 1, 041998.

[38] G. Verdier. Le principe des foils en un schéma où le basculement de la quille constitue une étape préalable essentielle. https://www.ouest-france.fr/vendee-globe/vendee-globe-foilsquilles-deux-poids-des-mesures-4588630, 2016.

[39] D. R. Verelst and T. J. Larsen. Load consequences when sweeping blades - a case study of a $5 \mathrm{mw}$ pitch controlled wind turbine. In Tech. Rep. Ris-R-1724(EN), Ris National Laboratory for Sustainable Energy3, 2010.

[40] J. Vierendeels, K. Dumont, and P.R. Verdonck. A partitioned strongly coupled fluid-structure interaction method to model heart valve dynamics. Journal of Computational and Applied Mathematics, 215(2):602 - 609, 2008. Proceedings of the Third International Conference on Advanced Computational Methods in Engineering (ACOMEN 2005).

[41] S. Volpi, M. Diez, and F. Stern. Multidisciplinary design optimization of a $3 \mathrm{~d}$ composite hydrofoil via variable accuracy architecture. In 2018 Multidisciplinary Analysis and Optimization Conference, page 4173, 2018.

[42] H. G. Weller, G. Tabor, H. Jasak, and C. Fureby. A tensorial approach to computational continuum mechanics using object-oriented techniques. Computers in physincs, Vol. 12, NO. 6, Nov/Dec 1998. Philip.

[43] Y. L. Young and S. Brizzolara. Numerical and physical investigation of a surface-piercing hydrofoil. Third International Symposium on Marine Propulsors (SMP13), Launceston, Tasmania, May, pages 58, 2013.

[44] Y. L. Young, N. Garg, P. A. Brandner, B. W. Pearce, D. Butler, D. Clarke, and A. W. Phillips. Load-dependent bend-twist coupling effects on the steady-state hydroelastic response of composite hydrofoils. Composite Structures, 189:398-418, 2018.

[45] W. Yu, V. Vitali, H. Dewey, Hodges, and H. Xianyu. Validation of the variational asymptotic beam sectional analysis (vabs). American Institute of Aeronautics and Astronautics Journal, 40, No. 10:21052113, 2002

[46] G.A. Zarruk, P.A. Brandner, B.W. Pearce, and A.W. Phillips. Experimental study of the steady fluid-structure interaction of flexible hydrofoils. Journal of Fluids and Structures, 51:326 - 343, 2014. 